\title{
Le lettere $D I$ e $A$ Cesarotti nella Biblioteca Nazionale di Parigi (CON Documenti inediti)
}

\section{Andrea Penso}

\begin{abstract}
L'articolo presenta due lettere di Melchiorre Cesarotti ritrovate dall'A. durante alcune ricerche condotte alla Bibliothèque Nationale de France su materiali manoscritti. La prima lettera è indirizzata a Voltaire, e riguarda la traduzione delle due tragedie Le Fanatisme, ou Mahomet e La Mort de César. Si tratta di una missiva già edita nel 1977 a opera di Theodore Besterman in Inghilterra nel quadro dell'edizione dei carteggi di Voltaire. Questo ritrovamento è passato però quasi inosservato nell'ambito degli studi di italianistica: l'articolo punta dunque a rilanciare l'interesse per questo fugace carteggio. La seconda missiva, che data al giugno 1803, è invece inedita e proviene dal fondo Custodi, inesauribile fonte di informazioni e curiosità. Il tema del secondo documento è molto diverso: Cesarotti comunica a Pietro Custodi di non poter prendere parte al suo progetto, la raccolta Scrittori classici italiani di economia politica, che iniziò a svilupparsi proprio nel 1803. Nonostante il rifiuto, il letterato padovano si ripromise di sollecitare qualche amico, esperto di economia, a fornire qualche contributo. Per meglio contestualizzare la seconda lettera, pertanto, l'A. si sofferma anche su un altro documento del fondo Custodi, vale a dire una missiva che l'economista veneziano Francesco Battaglia indirizzò proprio a Cesarotti, il quale l'aveva a sua volta incoraggiato a prendere parte al progetto di Custodi.
\end{abstract}

Lo sviluppo cui sono andati incontro in tempi recenti gli studi dedicati a Melchiorre Cesarotti testimonia come nell'ultimo ventennio la critica si sia orientata a una sistematica ripresa e rivalutazione della sua figura. Soprattutto a partire dal secondo centenario della morte (2008), il letterato di Selvazzano, che ebbe un ruolo non secondario nella cultura italiana ed europea del SetteOttocento, sta dunque ricevendo l'attenzione e l'importanza critica che merita. ${ }^{1}$

\footnotetext{
${ }^{1}$ Si segnalino almeno Gallo, Cesarotti da Padova a Selvazzano; Gallo, "Gli autografi cesarottiani”; Melchiorre Cesarotti nel bicentenario; Di Brazzà; Donà; Daniele (a cura di).
} 
Lattuale slancio degli studi cesarottiani ha messo in particolare evidenza due aspetti dello status quaestionis. Da un lato, la necessità di riscrivere alcune pagine della biografia letteraria di Cesarotti, alla luce dei risultati prodotti dalle più accurate e approfondite analisi storico-documentarie condotte negli ultimi anni. Dall'altro, l'urgenza di un'operazione di esame e rivalutazione per molte delle opere, che in vari casi necessitano di essere riviste con strumenti critici più solidi, in vista, com'è auspicabile, di edizioni filologicamente più attendibili.

A metà tra l'esigenza di un ripensamento della vicenda biografica e culturale di Cesarotti e il bisogno di operare una organica ristrutturazione delle sue opere, si colloca il monumentale volume di lettere scritte o ricevute da Cesarotti durante tutta la sua vita, e di cui oggi siamo a conoscenza. Le lettere, meglio di qualsiasi altra testimonianza pervenutaci, forniscono infatti una incredibile mole di dati e informazioni sulla biografia e sull'attività letteraria dell'intellettuale padovano. Il loro (ingente) numero e il loro essere perlopiù sparse in istituzioni di vari Paesi rendono evidente l'urgenza di un massiccio intervento per organizzarle in maniera sistematica, al fine di poter finalmente fruire di un moderno e aggiornato epistolario cesarottiano. Si deve soprattutto alle meritorie fatiche di Gilberto Pizzamiglio, Claudio Chiancone e Michela Fantato il più serio e recente tentativo di colmare le (enormi) lacune dell'unica edizione dell' Epistolario ad oggi disponibile, vale a dire quella curata da Barbieri a partire dal $1811 .^{2}$ Nei loro studi, cui rimando senz'altro, ${ }^{3}$ i tre hanno dimostrato quanto vasto e quanto vario sia il corpus dei carteggi

\footnotetext{
${ }^{2}$ Si tratta dei sei tomi XXXV-XL della raccolta Opere dell'abate Melchior Cesarotti padovano. 40 voll. Firenze: Molini, Landi e comp., 1800-1813.

${ }^{3}$ Pizzamiglio, Gilberto. “Alcune considerazioni preliminari." In Aspetti dell'opera e della fortuna di Melchiorre Cesarotti. Gargnano del Garda, 4-6 ottobre 2001. Vol. 1. A cura di Gennaro Barbarisi e Giulio Carnazzi. Milano: Cisalpino, 2002. 71-79; Fantato, Michela, e Claudio Chiancone. "'All'arrivo d'una mia lettera tutti sono avidi di sentirla': passato e futuro dell'epistolario di Cesarotti”. Giornale Storico della Letteratura Italiana 187. 617 (2010): 108-117. Fantato, Michela. "Per il censimento e l'edizione dei carteggi di Melchiorre Cesarotti. Parte I. Storia e fortuna dell'Epistolario." Studi sul Settecento e l'Ottocento 7 (2012): 121-130; Chiancone, Claudio. "Per il censimento e l'edizione dei carteggi di Melchiorre Cesarotti. Parte II. Storia di un lavoro ventennale." Studi sul Settecento e l'Ottocento 7 (2012): 131-138. Della Fantato si segnalano anche L'epistolario 'veneto' di Melchiorre Cesarotti: edizione critica e commento. Tesi di dottorato, Dipartimento di Italianistica e Filologia romanza, I ciclo n. s.. Università Ca' Foscari di Venezia, 2002-2003; "La dissimulazione onesta: il carteggio Cesarotti-Pagani Cesa." Quaderni veneti 42 (dicembre 2005): 119-177; "Melchiorre Cesarotti-Giuseppe Urbano Pagani Cesa: integrazione al carteggio (1781-1808)." Quaderni veneti 47-48 (gennaio-dicembre
} 
cesarottiani, scovando e catalogando documenti sconosciuti e lettere sparse. Ad oggi, dunque, il conteggio delle lettere di e a Cesarotti ammonterebbe a un totale di più di 1500 documenti non afferenti all'edizione Barbieri, custoditi in varie istituzioni italiane ed europee. È chiaro che siamo davanti a uno dei più notevoli repertori epistolari dell'epoca, quantitativamente oltre che qualitativamente; il fatto che esso non sia ancora consultabile nella sua interezza, oltre a far presagire che gli studi cesarottiani possono riservare ancora molte sorprese, rende evidente la necessità di un sistematico aggiornamento.

Nell'attesa che si concretizzi l'ambizioso progetto di una moderna edizione filologicamente affidabile dell'epistolario cesarottiano (e il lavoro di Pizzamiglio, Chiancone e Fantato è molto opportunamente orientato in questa direzione), desidero dar conto con il presente intervento delle mie ricerche condotte alla Bibliothèque National de France su materiali manoscritti, ${ }^{4}$ durante le quali ho avuto l'occasione di imbattermi in due lettere scritte da Cesarotti e in una a lui indirizzata, non incluse nell'edizione curata da Barbieri. Ritengo di un certo interesse la loro pubblicazione perché, pur non afferendo a nessuno dei corposi carteggi di cui siamo a conoscenza (le missive in questione testimoniano scambi molto fugaci), riguardano due momenti importanti (pur di differente rilevanza, come si vedrà) dell'attività culturale del letterato padovano. Si tratta, dunque, se non di scoperte interamente inedite, senza alcun dubbio di alcune significative tessere appartenenti a quel variopinto mosaico che è la vita di Cesarotti. Su questi due particolari momenti della carriera letteraria dello scrittore veneto la critica non si è mai soffermata se non cursoriamente: nei paragrafi seguenti si cercherà dunque di descrivere brevemente i carteggi e di collocarli nella vicenda biografica del letterato, tentando inoltre di chiarirne l'importanza.

2008): 143-188; Parleremo allora di cose, di persone, di libri. Lettere di Melchiorre Cesarotti a Francesco Rizzo Patarol. Venezia: Istituto Veneto di Scienze Lettere ed Arti, 2006. Sull'epistolario di Cesarotti cfr. ancora Gilardino, Sergio Maria. "Melchiorre Cesarotti". In Epistolari e carteggi del Settecento. Edizioni e ricerche in corso. A cura di Antonio Postigliola. Con la collaborazione di Gennaro Barbarisi e Nadia Boccara. Roma: s.n., 1985: 32-34.

${ }^{4}$ Materiali nei quali ho trovato una serie di biglietti inediti di Vincenzo Monti, insieme ad alcune varianti sconosciute di un sonetto ad Andrea Mustoxidi: di tutti intendo dar conto in un prossimo contributo. 


\section{Cesarotti e Voltaire}

L'interesse del primo documento è reso immediatamente manifesto dal nome del destinatario. La lettera è infatti indirizzata nientemeno che a Voltaire, personaggio di spicco nel panorama culturale dell'epoca che non ha certo bisogno di presentazioni. La missiva non è inedita: è stata infatti pubblicata nel 1977 a opera di Theodore Besterman in Inghilterra nell'edizione dell'intera corrispondenza di Voltaire. ${ }^{5}$ Nell'ambito degli studi di italianistica, questo ritrovamento è passato praticamente sotto silenzio, essendo stata la pubblicazione della lettera inserita in un'opera che per sua natura era ovviamente sbilanciata verso il punto di vista del francese. Se, dunque, per chi ha curato l'edizione dei carteggi di Voltaire quella di Cesarotti non è che una lettera tra le tante ricevute dal celebre poeta, così non deve essere invertendo la prospettiva: il fatto che il letterato padovano inaugurasse uno scambio epistolare con un personaggio così eminente (e con la cui opera letteraria si era confrontato) non può passare inosservato agli studiosi di italianistica, che devono invece incaricarsi di indagare le implicazioni e le conseguenze di questo carteggio. Ritengo dunque che la mancanza di studi che si siano soffermati, anche solo in maniera superficiale sul breve carteggio occorso tra Cesarotti e Voltaire costituisca una ragione sufficiente per riportare all'attenzione del pubblico degli italianisti questo importante documento, in vista di ulteriori e più approfonditi contributi.

Ma veniamo alla missiva. Da un punto di vista materiale, le carte sono prive di intestazioni e indicazioni chiare sul ricevente; le prove che confermano l'identità del destinatario derivano piuttosto dall'argomento (si parla delle traduzioni di due tragedie voltairiane) e dalla 'storica' provenienza. La lettera, scritta su quattro fogli di carta dalle dimensioni di $345 \times 270 \mathrm{~mm}$, è infatti inserita in una serie di documenti che riguardano appunto Voltaire, e che appartenevano in passato a Don Seymour de Ricci (1881-1942), bibliofilo francese che possedeva gran parte della corrispondenza voltairiana prima che venisse ceduta alla biblioteca. ${ }^{6}$ L'argomento della missiva è la traduzione che intorno al 1762 Cesarotti fece delle due tragedie Le Fanatisme, ou Mahomet e La Mort de César, scritte da Voltaire nel 1736 e rappresentate per la prima volta al pubblico rispettivamente nel 1741 e nel 1743 .

\footnotetext{
${ }^{5}$ Voltaire, lettera n. 12280.

${ }^{6}$ Le informazioni sulle lettere che si presentano in questa sede, inerenti la loro storia materiale e i loro vari possessori, sono state tratte dalle didascalie apposte alle stesse nel catalogo multimediale della BNF.
} 
Caratterizzata da una (lunghissima) captatio benevolentiae, in cui le lodi sperticate di Cesarotti al francese si mescolano ad alcune considerazioni sopra la poesia e la teoria della traduzione, la lettera si chiude con un accenno a "quelques discours" di accompagnamento alla traduzione. Il particolare mi autorizza a ipotizzare che la lettera in questione accompagnasse il dono del volumetto che Cesarotti fece stampare a Venezia nel 1762 presso la tipografia Pasquali, intitolato Il Cesare e il Maometto, tragedie del signor di Voltaire, trasportate in versi italiani, con alcuni ragionamenti del traduttore. I due Ragionamenti, uno Sopra il Cesare e l'altro Sopra il diletto della tragedia, si possono di fatto considerare come le prime formulazioni teoriche dell'estetica cesarottiana. In particolare, il Ragionamento sopra il diletto della Tragedia avrà una rilevanza notevole nel dibattito culturale dell'epoca. Il testo della lettera è il seguente: ${ }^{8}$

\begin{abstract}
Monsieur
C'est depuis long-temps, Monsieur, que je brûle d'envie de temoigner hautement aux gens de lettres et à vous tout le transport d'admiration, dont vos ouvrages m’ont frappé. Dés qu'ils me sont tombés entre le mains, j'ai crû reconnaitre le modele du Beau, que j'avais jusqu'alors recherché vainement, et je me suis aussitôt recrié: voila mon Apollon et mon Genie; je n'en veux point d'autre. Si les commentateurs avoient consacré leurs travaux à des auteurs tels que vous, Mathanasias n'aurait pas les rieurs de son coté. Je serai toujours persuadé que vous étes l'unique ecrivain pour qui c'est étre raisonnable que d'étre enthousiaste. Protée de la belle littérature vous prenez à votre gré toutes les formes, et dans chacune on reconnait toujours la méme divinité qui brille de toutes parts par de traits de lumiere et de feu dont il n'est pas permis d'ignorer la source. Mais ce qui vous rend à mes yeux le plus grand des Auteurs, c'est que vous avez rétabli la poesie et l'eloquence dans ses anciens droits presqu'oubliés d'instruire l'universe, et d'étre l'organ de la sagesse et de la vertu. Je révére en vous le Poéte de l'humanité; et n’en déplaise aux Orphées, et aux Amphions, je vous trouve bien plus propre qu'eux à operer les prodiges mysterieux de charmer les bétes, et d'attirer les bois. Que je chéris cet amour du genre humain qui dirige toujours votre plume: que
\end{abstract}

${ }^{7}$ Cesarotti, Melchiorre. Il Cesare e il Maometto, tragedie del signor di Voltaire, trasportate in versi italiani, con alcuni ragionamenti del traduttore. Venezia: presso Giambatista Pasquali, 1762.

${ }^{8}$ Nella trascrizione della lettera si è scelto di adottare un criterio ultraconservativo, riportando fedelmente anche tutti i refusi, che riguardano soprattutto l'accentazione. 
j'aime à voir la verité dans vos écrits tantôt s'armer des foudres de Jupiter pour écraser de grands préjugés, que les Philosophes ne font qu'indiquer froidement; tantôt se parer de la ceinture de Venus pour nous inspirer les plus belles et les plus douces vertus sociales; quelque fois en prenant l'air badin de Nomus purger la societé et la littérature de certains infectes, qui pour étre petits, n'en sont pas moins nuisibles, et les percer de traits d'autant plus mortels, qu'ils sont plus legers, et plus fins.

Ma foi, Monsieur, j'allois écrire une épitre et je m'apperçois que j'ai presque fait une ode. Ce n'est pas ma faute, si c'en est une. Le moyen de se defendre de l'enthousiasme, quand c'est de Voltaire qu'on écrit? Il faut donc vous dire, Monsieur, qu'ayant traduit il y a quelques années, deux de vos Tragedies pour les donner à declamer à une élite de jeunes-gens, je pren[d]s la liberté de vous les addresser, non comme un essai de mon faible esprit, mais comme un hommage publique qui j'aime à rendre à votre merite. L'étrange compliment, direz-vous peut-étre, que de presenter moi-méme à moi-méme tout défiguré. Je pourrais, Monsieur, pour ma justification vous étaler tous les lieux-communs des Traducteurs: mais je veux bien vous en faire grave. Je pardonne à la diversité du langage et de la versification tous les obstacles qu'elles m'ont opposés, pour ne me plaindre que de vous. Avérez, Monsieur, que ce n'est pas une petite affaire que d'étre aux prises avec un modèle tel que vous. Vous mettez au méme point les Traducteurs, et les ecrivains: vous desesperez à la fois les uns et les autres. On est bien plus à son aige avec la plûpart des auteurs. Il est permis quelque fois de gâter son texte sans que personne ait lieu de s'en plaindre: cela ne tire point à consequence. Sentiment pou sentiment, l'un vaut bien l'autre. Mais comment prendre des mémes libertez en trasportant d'une langue à l'autre des ouvrages d'une beauté de style si achevée qu'il faut se recrier à tout moment avec vótre Pangloss, mais peut-étre avec phis de raison que lui, tout est bien tout $\underline{\text { est }} \underline{\text { au mieux, tout }}$ est on ne peut pas mieux. D'autre part comment vous traduire au pied de la lettre? En tachant de rendre vos mots, j'aurais affaiblis vos traits. J'ai taché de mon mieux de m'écarter également de ces deux facheuses extremitez. Si vous ne vous deplaisez pas tout-à-fait dans cet habillement étranger, j'en serai ravi; si j'ai manqué mon but, je m'en consolerai aisément, parce que j'espere que cela tournera moins à mon desavantage, qu'à votre gloire. J'ai joint à ma Traduction quelques discours dans celui sur Cesar, vous verrez que je me suis fait une difficulté que j'ose proposer à vous méme. Je l'aurais dissimilée, si je 
vous respectais moins. Instruisez-moi, redressez-moi: je serai plus charmé de vos corrections, que des éloges des autres.

Vous verrez aussi un essai sur l'origine et les progrês de l'art poetique. Si l'impartialité et la franchise que j'ai puisée dans vos écrits a le bonheur de vous plaire, je me croirai assez dédommagé des reproches que je ne peux manquer de m'attirer de la part du vulgaire des critiques. Votre approbation est un passeport pour le pays de la posterité qui vous appartient par tant de droits. Ce serait ridicule pour moi que d'y pretendre. Mais je n'ose y aspirer que comme à la suite d'un grand Prince qui prend possession de son domaine.

Je suis, Monsieur, avec tout le respect que les rangs usurpent sur les talents

Le plus zelé de vos admirateurs

L'abbé Cesarotti

Dell'importanza del Ragionamento sopra il diletto della Tragedia si era d'altra parte accorto lo stesso Voltaire, come mostreremo tra poco. Nell'edizione dell'epistolario cesarottiano curata da Barbieri è infatti possibile ritrovare la lettera che il francese indirizzò a Cesarotti per ringraziarlo dell'omaggio tributatogli (55). La missiva che si pubblica in questa sede chiude pertanto il cerchio del fugace carteggio occorso tra i due, e aiuta a comprendere le ragioni della risposta che Voltaire indirizzò all'italiano. Il testo della responsiva edita da Barbieri è il seguente:

10 janv. 1766, au Chateau de Ferney

\section{Monsieur}

Je fus bien agréablement surpris de recevoir ces jours passés la belle traduction que vous avez daigné faire de la Mort de César, et de la tragédie de Mahomet. Les maladies qui me tourmentent, et la perte de la vue dont je suis menacé, ont cédé à l'empressement de vous lire: j'ai trouvé dans votre style tant de force et tant de naturel, que j'ai cru n'être que votre faible traducteur, et que je vous ai cru l'auteur de l'original. Mais plus je vous ai lu, plus j'ai senti que si vous aviez fait ces pièces, vous les auriez bien plus mérité d'être traduit. Je vois en vous lisant la supériorité que la langue italienne a sur la nôtre; elle dit tout ce qu'elle veut, et la langue française ne dit que ce qu'elle peut. Votre discours sur la tragédie, Monsieur, est digne de vos beaux vers; il est aussi judicieux que votre poësie: il me parait que vous découvrez d'une 
main bien habile tous les ressorts du cœur humain; et je ne doute pas que si vous avez fait des Tragédies, elles ne doivent servir d'exemples, comme vos raisonnements servent de préceptes. Quand on a si bien montré les chemins, on y marche sans s'égarer. Je suis persuadé que les italiens seraient nos maîtres dans l'art du théatre, comme ils l'ont été dans tant de genres, si le beau monstre de l'opera n'avait forcé la vrai Tragédie à se cacher. C'est bien dommage, en vérite, qu'on abandonne l'art de Sophocles et Euripides pour une douzaine d'ariettes fredonnées par des eunuques. Je vous en dirais davantage si le triste état où je suis me le permettait. Je suis obligé même de me servir d'une main étrangère pour vous témoigner ma reconnaissance, et pour vous dire d'une petite partie de ce que je pense; sans cela, j'aurais peut être osé vous écrire dans cette belle langue Italienne qui devient encore plus belle sous vos mains. Je ne puis finir, Monsieur, sans vous parler de vos ïambes latins, et si je n'y étais pas tant loué je vous dirais que j'ai cru y retrouver le style de Terence. Agréez, Monsieur, tous les sentimen[t]s de mon estime, mes sincères remerciemen $[\mathrm{t}] \mathrm{s}$, et mes regrêts de n'avoir point vu cette Italie à qui vous faites tant d'honneur. J'ai l'honneur d'être ave ces sentimen $[\mathrm{t}] \mathrm{s}$,

\section{Monsieur}

Vôtres très-humble et très obéissant serviteur VOLTAIRE gentilhomme ord.re de la chambre du Roy

Come si può notare, dopo i ringraziamenti e i convenevoli di rito, nella sua risposta Voltaire indugia volentieri a parlare del "discours sur la tragédie", segno che di quelle riflessioni doveva aver colto l'importanza. Oltretutto, sono assenti chiose sull'altro Ragionamento: sembra perciò che il letterato francese sia stato mosso da sincera partecipazione nel commentare lo scritto sul diletto della tragedia, e non da ragioni di opportunità e galanteria. Chiude la lettera di Voltaire un suo apprezzamento per i giambi di Cesarotti, prova che il volumetto stampato da Pasquali fu tra le mani del francese. In quell'edizione, leggiamo infatti i giambi che cominciano: "Favete linguis, atque animum huc advortite; / Venio a bicipiti, praeco Musarum, jugo, / Apollinaris Curiae sententiam / Super Poetis Tragicis ad vos deferens" (8). In chiusura, si rilevi la mancanza, oltre che dell'intestazione, anche della data della lettera. 
La mia ipotesi, suffragata da altri documenti dell'epistolario curato da Barbieri, è che la missiva sia stata redatta da Cesarotti verso la fine di febbraio e i primi di marzo del 1765 . In una lettera collocabile cronologicamente intorno al 19 marzo 1765 e diretta all'abate Taruff, Cesarotti scrive: "puisque vous me rappelez le célébre Voltaire, je serai ravi de savoir si on lui a rendu de ma part une lettre avec un exemplaire de ma traductions de ses deux Tragédies dont M.r Goldoni s'etoit chargé dans son voyage" (Opere 26). Fu dunque il drammaturgo veneziano a fungere da corriere per la consegna del plico cesarottiano. Il viaggio di Goldoni qui menzionato non sarà, per ovvie ragioni di datazione, quello originale in Francia del 1762, bensì piuttosto una delle sue visite programmate a Voltaire, che deve essere avvenuta in tempo utile perché il francese avesse modo di ricevere il pacchetto e compilare la sua risposta il 10 gennaio dell'anno seguente. Voltaire scrive infatti di aver avuto le traduzioni "ces jours passés", ed è quindi plausibile che, al netto dei tempi tecnici per le consegne postali, la lettera, partita quasi certamente dall'Italia proprio in un momento compreso tra febbraio e marzo del 1765, abbia percorso un itinerario particolarmente complicato. Ammesso e non concesso, ovviamente, che in effetti il francese non dovesse fare ammenda per un considerevole ritardo responsivo, o che Goldoni non avesse rinviato di quasi nove mesi la visita da cui dipendeva la consegna.

Alla luce di queste considerazioni, una domanda sorge comunque spontanea: per quale ragione Cesarotti aspettò tre anni (si ricordi che le traduzioni si stamparono a Venezia nel 1762) prima di scrivere a Voltaire per mandargli una copia delle sue versioni? La risposta, secondo me, va cercata in una sorta di timore reverenziale che il letterato padovano doveva nutrire nei confronti dell'astro francese. Se si guarda al tono della missiva scritta da Cesarotti, si scorgono senza troppa difficoltà tantissime frasi che quasi sconfinano nell'adulazione e le lodi talvolta sembrano percorrere delle tangenti che alla lunga risultano eccentriche rispetto allo scopo del messaggio. Viceversa, la risposta di Voltaire è molto scarna, certamente cordiale e sincera, ma fondamentalmente priva del trasporto riscontrabile in Cesarotti. È segno di un rapporto sbilanciato, in cui chi omaggia pare consapevole di occupare una posizione subordinata. Il lasso di tempo trascorso dalla pubblicazione delle versioni delle tragedie all'omaggio rivolto al loro primo autore potrebbe dunque nascondere la volontà di Cesarotti di acquistare maggior credito e maggior popolarità prima di tentare di stabilire un contatto che doveva sembrargli in qualche modo audace. Tale ipotesi potrebbe essere suffragata dal fatto che a partire dal 1763 Cesarotti aveva iniziato la traduzione dell'Ossian, vale a 
dire di quell'opera che gli avrebbe dato la fama e che aveva avuto immediatamente un buon riscontro. Il letterato padovano dovette credere di aver acquisito credito sufficiente nel 1765 per avere l'ardire di scrivere a un personaggio così importante. In altre parole, sembra lecito ipotizzare che, solo dopo una più ampia affermazione sulla scena culturale, Cesarotti si sia sentito in diritto di scrivere a Voltaire. Valga questo episodio come testimonianza della continua evoluzione che conobbe la carriera letteraria di Cesarotti.

\section{Alle radici di una (auto)esclusione: Cesarotti e Pietro Custodi}

La seconda lettera cesarottiana che intendo presentare è invece inedita ed è conservata in quello sterminato repertorio di informazioni e documenti che è il fondo Custodi, passato dalle mani del suo proprietario a quelle dei Costa de Beauregard nel 1842 (anno della morte del Custodi), fino all'acquisizione definitiva da parte della Bibliothèque Impériale nel 1867. La missiva è indirizzata proprio a Pietro Custodi:

Mio Sig.re

Pad.[ov]a 23 aprile 1803

Bench'ella sia illuso dalla sua gentilezza nel caratterizzarmi con titoli così speziosi, non si inganna però nel credermi vivamente interessato per la gloria d'Italia e per quelli che confluiscono a propagarla e promuoverla. Ho perciò accolto con somma compiacenza il prospetto della sua Raccolta egregiamente concepito ed espresso. Se la natura de' miei studi non mi permette di coadjuvar direttamente un progetto di cui conosco tutta l'importanza, mi farò almeno un pregio di sollecitar qualch'amico dotto ed esperto in quegli argomenti a prendervi parte e col favore e coll'opera. Il di lei nome mi era noto vantaggiosamente fin dai principj delle peripezie italiche. Ora questo testimonio della sua graziosa propensione unisce alla stima la gratitudine e mi induce a protestargliela col più ingenuo compiacimento

Salute, onore e prosperità

Cesarotti

Per quanto riguarda l'aspetto materiale della lettera, è da notare, rispetto alla precedente, il tono sbrigativo e il ductus poco sorvegliato con cui è scritto il 
messaggio: è vero che sono passati moltissimi anni dalla lettera a Voltaire, e che a questa altezza cronologica Cesarotti era piuttosto anziano, ma ciò non pare una giustificazione plausibile per la sbrigatività con cui sembra essere stata redatta la missiva. La formula di commiato è addirittura costituita da tre lapidarie parole, "Salute, onore, prosperità" seguite dal solo cognome "Cesarotti". Per quanto riguarda la grafia, poi, alcuni passaggi sono stati di difficile interpretazione," proprio a causa della trascuratezza con cui sono scritte le parole. Ad esempio, la concessiva a metà testo è introdotta da un "Se" evidentemente aggiunto in un secondo momento, con l'articolo "La" già presente sul foglio. È il sintomo di una stesura frettolosa e in qualche modo poco interessata e partecipe, ancorché sempre cordiale (non vengono risparmiate lodi al destinatario in chiusura). L'argomento della conversazione comunque è immediatamente chiaro: Cesarotti comunica a Custodi di non poter prendere parte al suo "progetto" pur riconoscendone l'importanza. Il progetto editoriale in questione è la raccolta Scrittori classici italiani di economia politica, ${ }^{10}$ che proprio nel 1803 si avviava a prendere forma. $\mathrm{E}$ necessario dunque supporre che questa lettera sia stata preceduta da un messaggio del Custodi, che doveva aver chiesto all'ormai affermato letterato padovano di avere un ruolo attivo nel suo piano di lavoro. Cesarotti declinò, visto che la natura dei suoi studi lo portava in altre direzioni, promettendo però di sollecitar qualche amico dotto in materia di economia. Cosa che in effetti fece: tra questi amici ci fu ad esempio certamente l'economista veneziano Francesco Battaglia. Una sua lettera scritta da Padova il 30 giugno 1803 e diretta a Cesarotti ci informa del fatto che in quel periodo Battaglia stava riesumando alcuni suoi scritti economici, su sollecitazione del professore padovano, per poterli offrire col suo tramite a Pietro Custodi, allo scopo di includerli nella Raccolta. La trascrizione della lunga missiva di Battaglia (la cui riproduzione si pubblica in appendice) non è priva di interesse, per comprendere cosa iniziò a svilupparsi intorno al primo contatto epistolare occorso tra Cesarotti e Custodi: ${ }^{11}$

Pregiatissimo Cesarotti

Padova 30 giugno 1803

\footnotetext{
9 Particolari grattacapi ha creato ad esempio la lettura di "coadjuvar", lemma cesarottiano che viene adottato anche altrove, qui scritto nella variante con la “ $\mathrm{j}$ ”.

${ }^{10}$ Pietro Custodi (a cura di). Scrittori classici italiani di economia politica. 50 voll. Milano: G. G. Destefanis, 1803-1816.

${ }^{11}$ Anche questa lettera è conservata alla BNF: è contenuta nel Ms. Italien 1547.
} 
Lasciando d'esaminare se in nessun tempo della mia vita io avessi potuto intraprendere la difusione [sic] di qualche scritto, difficilmente avrei potuto farlo anche per le mie occupazioni nel servire la fu Patria mia che mi hanno costato meditazioni continue, vigilie, inquietudini, amarezze d'ogni genere, e posso dire una robusta salute: ed ella che sa i fatti miei lo conosce. Ma sentendola vivamente impegnata per favorire un'amico [sic] suo di Milano occupato nella buona riuscita della raccolta che si vuole stampare d'autori italiani che hanno scritto di Politica Economia, così le fo tenere un grosso volume di sedici pagine in dodici che non sarà noto probabilmente agli editori di Milano, e che nacque così. Leggendo, anzi meditando sopra l'opera dello Smith a ragione celebratissima, convinto di quanto disse sopra uno dei mezzi creduto dalla maggior parte attissimo a promuovere le arti, ed a perfezionarle, ho voluto tentare se si poteva con assai meno tempo lui provare lo stesso assunto. Mi sono anche immaginato d'esserci riuscito, avendo in oltre fatto dei cenni sopra altri oggetti che mi si presentavano all'intelletto per la loro stretta attinenza con l'assunto principale: in seguito mi venne in testa di fare stampare quanto avea scritto.

Ma poiché Ella mi ha raccomandato di darci tutto ciò che avessi potuto avere di stampato, e anco di non stampato sopra tali argomenti, penso di farle avere anche quattro memorie, che nel fu Senato Veneziano si nominavano scritture, sopra tre punti importanti: una sulle Cartiere, due sul commercio delle lane interno, ed esterno, ed una sui Lanifizi, le quali dettero occasione a vivissime discussioni. A dire il vero per renderle accomodate alla raccolta che si sta formando bisognava rifarle, levandoci tutto ciò che si riferisce alle circostanze che le hanno fatte nascere, ma la cosa mi saria riuscita lunga, e faticosa, avendo il piacevole ozio in cui mi vivo da tempo non breve arrugginite le mie facoltà intellettuali. S’è in esse dovuto parlare delle mani intermedie, ma rileggendole osservai una omissione, ed è quella di provare che quando pure un governo con pessimo consiglio, e con una violenza proporzionata alle difficoltà fosse venuto a capo di toglierle non si sarebbero per questo né tolti gli effetti di esse, ne migliorata la condizione dei consumatori. Ho dunque creduto in una nota brevissima farci questa piccola aggiunta, come pure un cenno sopra qualche contradizione che è di sola apparenza.

Eccola dunque servita come ho potuto lasciandola poi in totale libertà di spedirle, o di non le spedire a Milano, pregando lei solo (spedendole) di non dire d'averle da me ricevute. Se poi a Milano non ne faranno nulla, il 
mio amor proprio non se n'offenderà punto: mentre (dico io) sono proprio divenuto Filosofo nelle cose di questo mondo, sebbene non sia dello stesso parere la nostra cara, ed amabilissima presso cui passiamo dell'ore assai deliziose, la quale ha deciso che la mia pretesa filosofia non sia che sola, sola stupidezza. Ella si conservi in buona salute, e faccia viso torto alla minacciata disgraz[ia] ad'uno degli ornamenti dell'ameno suo Salvagiano. E senza più mi creda

Di lei

Ammiratore, ed amico

Franeeseo Battagtia

Il progetto di Custodi doveva quindi aver colpito Cesarotti se, come mostra la lettera di Battaglia, si attivò per coinvolgere qualche amico più esperto; ma non poi così tanto, vista la comunicazione dell'impossibilità a prendervi parte, avvenuta con le modalità di "distacco" che abbiamo più sopra rapidamente descritte. Quanto a Battaglia, poco si conosce dei suoi studi e delle sue prime iniziative politiche. Le testimonianze della maturità restituiscono invece l'immagine di un uomo molto impegnato sul versante politico ed economico di Venezia. La sua attività politica, condotta fra il 1770 e il 1790 ricoprendo diverse cariche dell'amministrazione della Repubblica, attesta dell'attenzione particolare rivolta ai dibattiti economici, che lo resero protagonista in Senato di vivaci polemiche sui problemi commerciali che lo Stato veneto si trovava a dover fronteggiare in quei decenni. Battaglia fu sostenitore di una maggiore libertà economica contro i numerosi monopoli, che la tradizione legislativa veneta aveva imposto da tempo alla gestione del paese. Più volte eletto nel Collegio dei Savi, cui era affidata la direzione politica generale della Serenissima, Battaglia affiancò la sua condotta in campo economico con un ponderato atteggiamento politico. Autorevole rappresentante in Senato del gruppo dei patrizi inclini ad una nuova politica più aperta alle moderne esigenze, resesi evidenti anche nella stanca vita politica della Serenissima, fu promotore di proposte per un rinnovamento interno, inquadrate in una serie di attacchi portati alla politica economica della Repubblica, la quale era ormai alle soglie della sua rovina definitiva. Fautore di una politica di completa remissività al volere della Francia nelle vicende che segnarono l'ultimo decennio del XVIII secolo, Battaglia fu oggetto di alcune accuse di tradimento che progressivamente insospettirono gli inquisitori di Stato, stanti anche i suoi rapporti personali con Napoleone. In 
effetti, quella Terraferma che dal 1796 era stata affidata alla sua giurisdizione poteva considerarsi ceduta alla volontà e alla discrezione del Bonaparte e delle armate francesi, complici anche la paura e la debolezza che regnavano a Venezia. Il trattato di Campoformio mise fine all'attività politica di Battaglia e all'indipendenza politica veneta. Rifugiatosi a Padova, Battaglia visse triste e ritirato: la lettera al Cesarotti, scritta da Padova il 30 giugno 1803, è l'ultima sua testimonianza in nostro possesso. I saggi di Battaglia, elaborati a partire dall'opera dello Smith e attinenti i mezzi di incoraggiamento delle manifatture, il commercio della lana, le industrie della seta e della carta, non trovarono infine spazio nella monumentale silloge del Custodi perché il Battaglia si rifiutò di ritoccarli, essendo da troppo tempo 'arrugginito' proprio a causa dell'ozio forzato cui era stato relegato dopo il trattato di Campoformio. ${ }^{12}$

Lo scopo di questo contributo era quello di presentare alcuni tasselli della complessa e per certa parte ancora poco conosciuta vicenda biografica di Cesarotti. Gli spunti per futuri interventi che prendano le mosse da queste due lettere sono molteplici: come venne tradotto Voltaire da Cesarotti? ${ }^{13}$ Quali sono le caratteristiche delle due versioni, che di fatto costituiscono la palestra per la successiva tradizione dell'Ossian? E ancora: in che rapporti stava Cesarotti con l'ambiente culturale milanese, di cui Custodi era un non secondario rappresentante? Su queste e su altre rilevanti questioni mi ripropongo di diffondermi maggiormente in prossimi contributi, approfondendo le ricerche e allargando il raggio dell'analisi storico-documentaria.

FWO - Fonds Wetenschappelijk Onderzoek / VUB - Vrije Universiteit Brussel

${ }^{12}$ Le informazioni sulla vita di Battaglia sono state tratte dalla pagina a lui dedicata nell'enciclopedia Treccani e consultabile al link http:/www.treccani.it/enciclopedia/ francesco-battaglia_(Dizionario-Biografico).

${ }^{13}$ Cfr. intanto Frassineti. 


\title{
APPENDICE
}

I.

Lettera a Voltaire, febbraio/marzo 1765? (entro il 10 gennaio 1766)

\author{
Howjiener
}

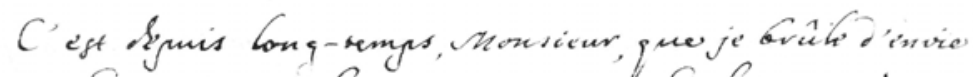
20 temoiquer hautement aux gins $\partial 2$ Cefere's of a' vous

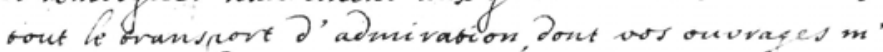
ont frappé. 'De's qu'its me sout rombe's e'utro les mains $j$ 'ai un veconnoibre le moiele on Beare que $j$ 'avois iusqu' a fors vicherché vaiminent, et je hne suis augitôt vecrié voila mon tor hlon ét mon genie; jè n'en veux roint d'antre. Sa les Commentakeurs avoient corjacré Kurs trawaux a' Des aubeurs rels que wous chathanasias n'auvoit gas les vicuss be'sol coté. He sarai rou-

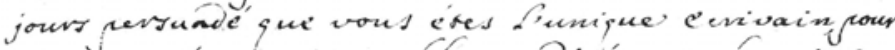

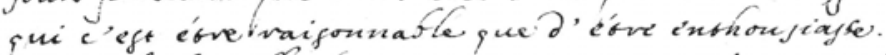
wroted of la belle listerabuth vous mene? a' votn'gné routed les formes, it dans chacune on reconnoit roujous La méme oivinité qui orith de toubes carts par detraits is fumieve et be fen, Jont if n'egt cas cermis?" iqnorer a source. Mais ce qui vous vind a' nes yeuse

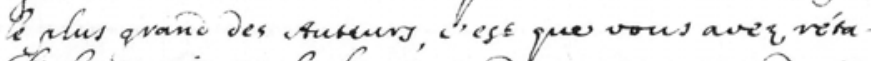
ofi la joogie et feloquince dans ses anciens droits

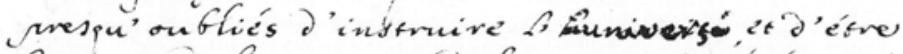
Porgane de la raqujge et de la verbu. Je vévére ex vous le Porte de' humanité; et n'en ojélaige aux Or. 
chées, et aux Amphions, je vous trouve bien cales propor

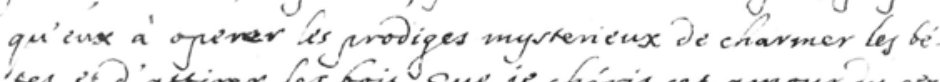

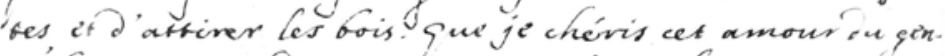
re' humain qui divige ronjours vótre clume' que j'ar me a'voir la verive dans vos écrios tanofots armer ofs foures $\partial e$ Inwiter pour écraser de grands préjug és su

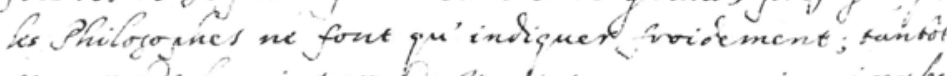

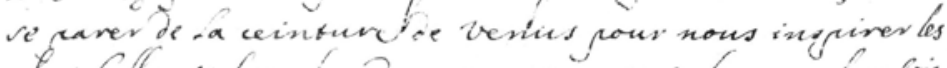

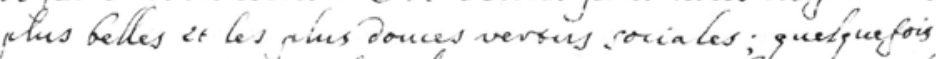

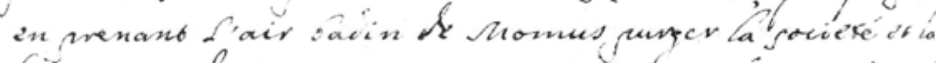
litkerature of cerbains ingectes, qui cour etres pebits,

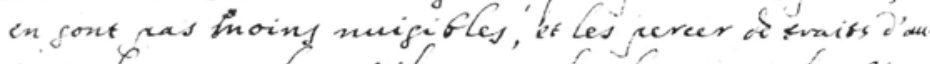
tant glus morkels, pu'ils pont plar capers et plas fins.

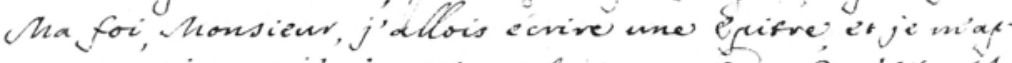
pergois que $j$ 'ai prespue fait une bov. Ce n'egt pas ma facte si c'en egt une re moyen de se defenove de Fenthousiasme, quand c'est de Voftain' pu'on eint.

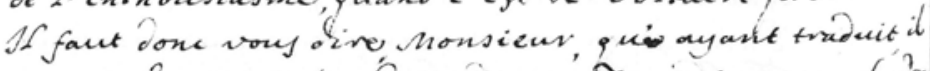

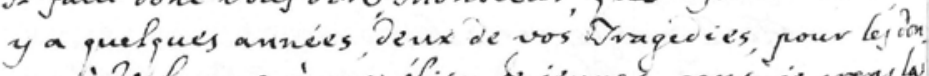
ner d' Diclamer a'une éliter de jeuncs-gens, je prexila liferré \&o vous les arejser, non comme un efsai de now foible zspit, mais comme un hommage, nuflique git 
j'aime a' vendr' a'votre menide. C'étrange comn lime'nt, diviz-vous pent-etre, que depresenter mov-míme a' moi-mémo rout séfiguré. 'e pourvois monsicur gour ma justification vous ćtater ici toug les fieux-commons Des Jiaduckems: mais je venx fien voug in farine graw.

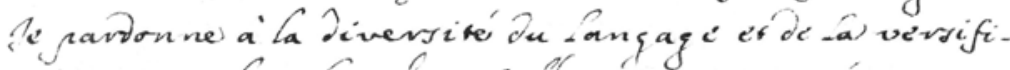
cation boug les ofstacies qu'elles min ont ogoséx, gour

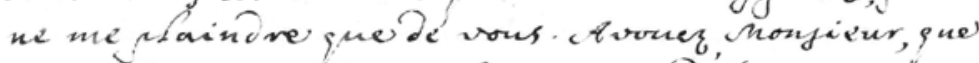

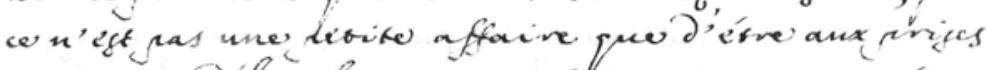
ave un modele tel pue vous. Thous mester ase méme

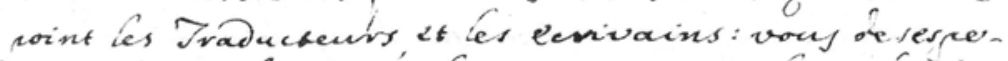

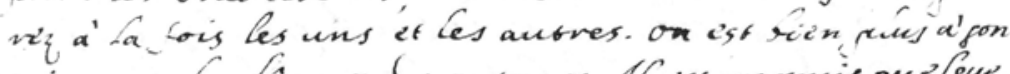

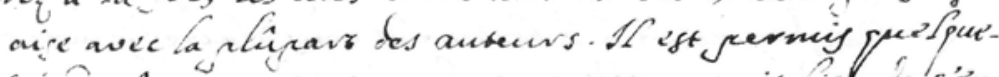
fois 22 , âker gon kexter sans que pergonne ait hick tés'én plainove cela ne bive point a consépenceldentiment

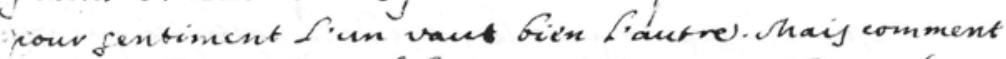

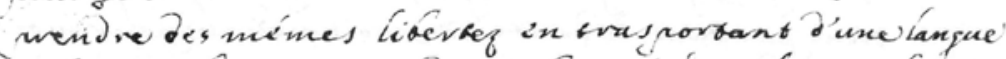

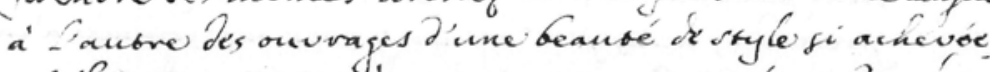
qu'ilfout se viovier a tout monent ave' vótre sangloss

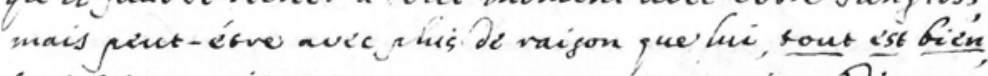
fout egt an mienx, tout egt on ne recie pay mitax. D'autro part comment vouy svaduin au pied de fa Cute' 'n rachant de 


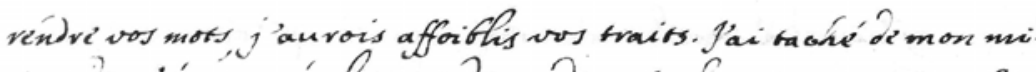

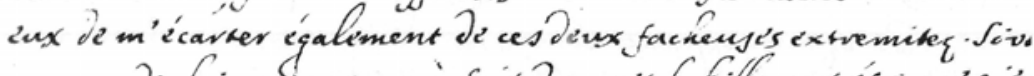

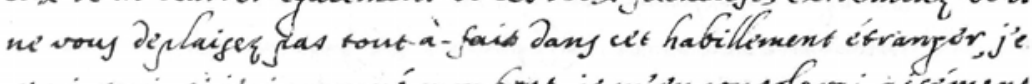

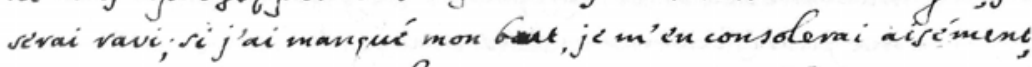
carce que' i'esperer qué cela sournera moins a'mon de'savantage: qu'a votre qioive! J'ai jont a' nia Draduction quetgues Discours

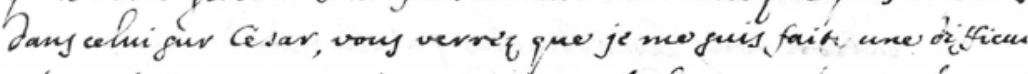

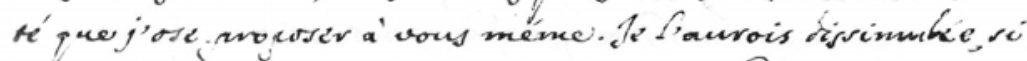
$j 2$ ous respectois moins. Instruige moi, vedryser-moi : $j$ é

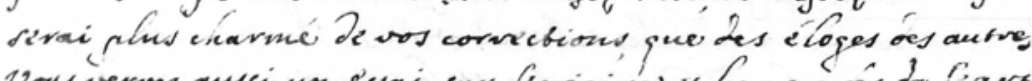
Voug verveg angi un cesaci sur forigines et les prog vés do favt

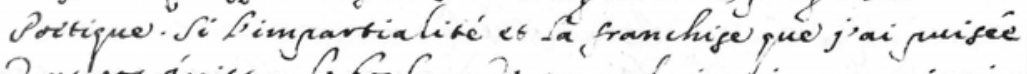
Dang wor écrits a le fonkeur $\partial 2$ voug flaive, je mer croinaciay

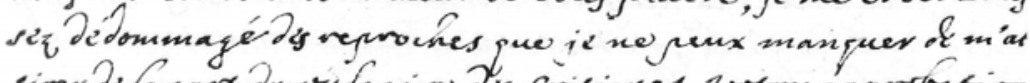

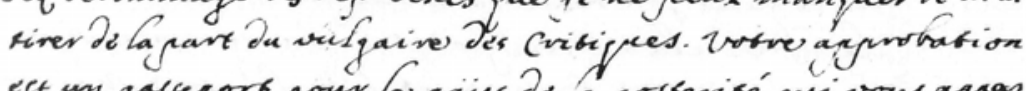

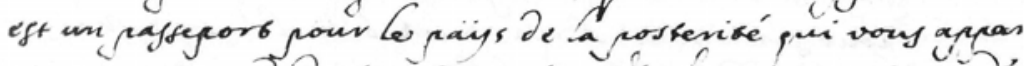

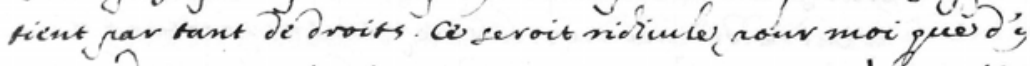
pretender, mail je n' ose yaspirer pue commo a' id suite

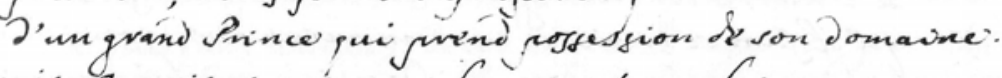
De guis, hongienr, wéc sout lo vegreet ques les vangs ugurgen

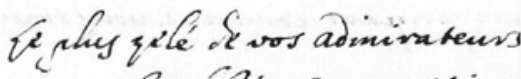

$$
\begin{aligned}
& \text { Caobe' Cesarobi. }
\end{aligned}
$$


Més

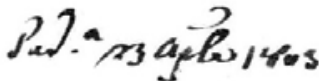

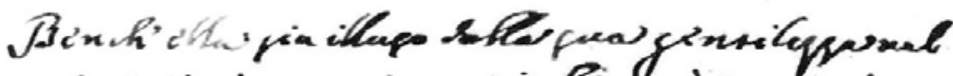

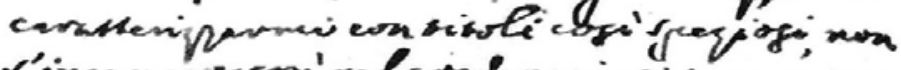

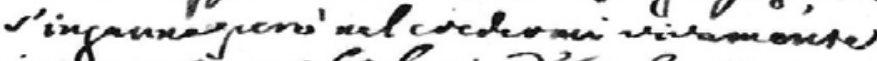

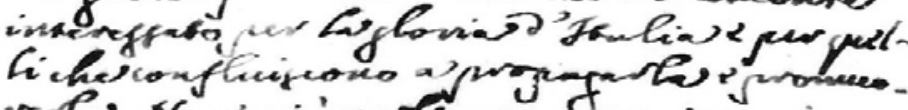

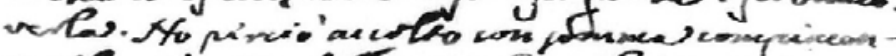

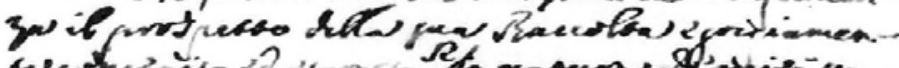

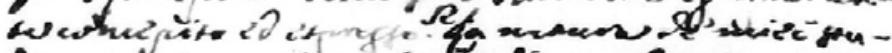

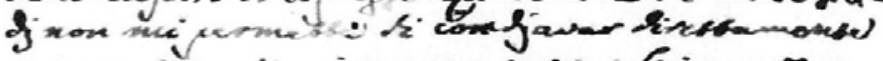

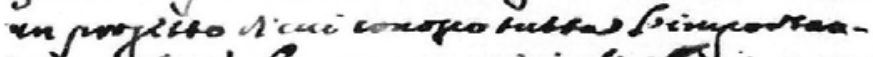

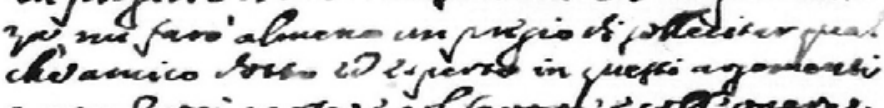

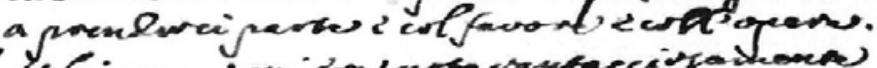

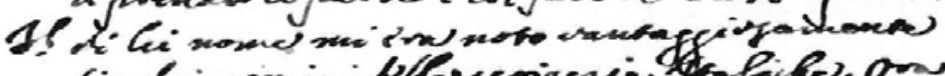

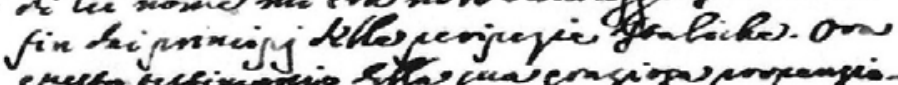

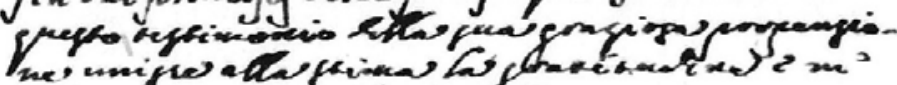

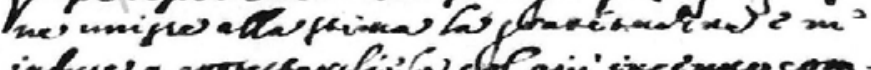

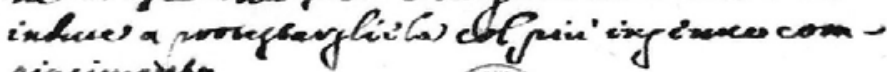
vicucioverter

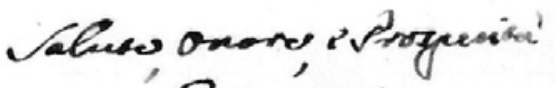
Ceganopec 


\section{III.}

Lettera di Francesco Battaglia a Cesarotti, 30 giugno 1803

Bregeation Lefavoti Babou so Riugnos sos

Sajciando d'ejaminare se in nepun tempo della mia ista co avefli vo tuto intrapenderve la difujione ri qualche scilte, ditticimente avvei votuto favlo anche per le nie oceuparioni nel sevrive la fo batria mia che mi hanno costato mestarioni continue vigilie, inquietuctini, amaverre $\partial$ 'ognigenere, a.

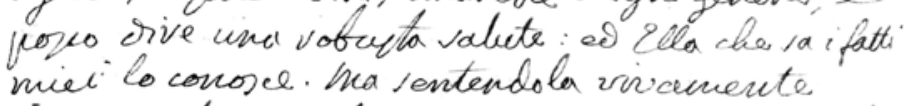
impregnate per favonive un amico suo di Bnilano oceupato vella buoma niejita della vano th che si vole tampere daton Atalianiche hamo scritte di folition Ronomia coi'le fo Leneve un grow wolume divedici uagene in dockich non sova' noto wobabilmente agli esitor ci vilare, e che nacque cori. Eggendo, anzi meditando. sofua Popera dello mith a vagione celefratipima cominto ir quanto dife sorauno deimers. creduto dalla maggios narte atticino on wommonere le avt, el a per, ferionarte, ho voluto tentare se rivotera con a paimen tempro di len provare bo jterso arranto. ne somo anche immaqinato d'eprevinicescito, 
avembo in ottre fotto deicenvi vorva alts oggetli che mi si presentaveno all 'intellesto per la lovo trello altinenra con P'apsanto principale: in requito mi veme in Cela or fare tamirave quantes area sintto.

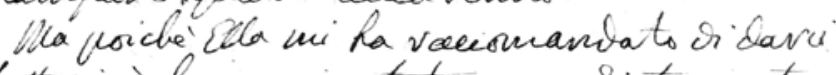
tulto cio'che avefi potuto avere si tampato, es ano or non tampats sowa tali arfonenti, pens rifarle acreve avele quattro memovie, che nel fu Senato Veveriano-vinominavano scritture, sopwa the punti importacti: una sulle Cartieve, due sul canercio deche lane. interns, eo efterno, ed una vai tanifizi, le quali detters oceajione a vivisine dejaysioni. a dive il vero per vendevle anomodate alla vacolta che vi ta formando fijognawa nifarle, levandori tutto cis che si viferiyce afle aivoitanze che le hamo fatte najcere, ma la core mi sovio vicfuta lunge, \& faticora, avends il fiacarole oris in cui mi zivo da tempo non overe. arvuginite le mie facolto intellettual. 'é' in eve dourto pavtave delle mani intermerio, 
ma vileggendole orevrai una onificone, ede'quell gi quarave che quarbo pure un foverno con pelsimo conjigho, e con una violenra urovorio. vata alle ditficolta' fore venuto a capo di toglievle non sivavebbero per queito ve toltigle effett is epe, we migliovata la condizione dei comumaton. fo diunge eveduto in una rota orerisioma. farci queta vius haggiunts, care pure un cems

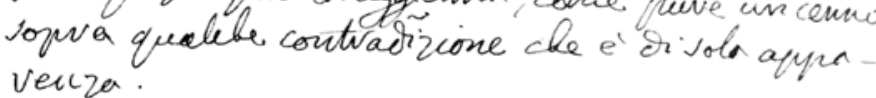

Sccolo dunque verita come ho potuto lavian-

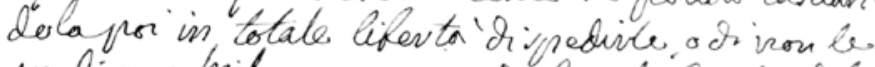
spedive a hilano, veyando le volo (spedendole) inon cive d'avente da me nicurete. Posoia Milo no non he faceqero null, ince amor promi wou 1 n'offendera' punto: mentre gico io) sono propino tivenuto vilos fo velle cose siqueito mondo, reotene non sia dello tejto pareve la notva cava, ef amabiliftima amiea prepto cu nopiamo dell'ove afraidelizioe, la quale ho decifo che la mia preteja Rilozotia $n$ on via che

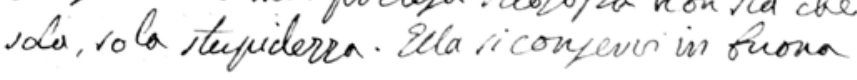


Salute, e facia vico tooto alla minaciata disgrar ad uno deglioonamenta delíameno suo Salvagiano frensa juirmi creda

$$
\text { Diser }
$$




\section{Opere Citate}

Barbarisi, Gennaro e Giulio Carnazzi (a cura di). Aspetti dell'opera e della fortuna di Melchiorre Cesarotti (Gargnano del Garda, 4-6 ottobre 2001). Milano: Cisalpino, 2002.

Cesarotti, Melchiorre. Il Cesare e il Maometto, tragedie del signor di Voltaire, trasportate in versi italiani, con alcuni ragionamenti del traduttore. Venezia: presso Giambatista Pasquali, 1762.

. Opere dell'abate Melchior Cesarotti padovano, 40 voll. Firenze: Molini, Landi e comp., 1800-1813.

Chiancone, Claudio. "Per il censimento e l'edizione dei carteggi di Melchiorre Cesarotti. Parte II. Storia di un lavoro ventennale". Studi sul Settecento e l'Ottocento 7 (2012): 131-138.

Custodi, Pietro (a cura di). Scrittori classici italiani di economia politica. 50 voll. Milano: G. G. Destefanis, 1803-1816.

Daniele, Antonio (a cura di). Melchiorre Cesarotti. Atti del convegno di Padova (4-5 novembre 2008). Padova: Esedra, 2011.

Di Brazzà, Fabiana. "La corrispondenza epistolare tra Melchiorre Cesarotti e Lavinia Florio Dragoni”. Studi veneziani 55 (2008): 391-478.

Donà, Chiara. Selvaggiano. Un letterato, un luogo e la sua storia. Padova: Grafiche Turato, 2008.

Fantato, Michela, e Claudio Chiancone. "All'arrivo d'una mia lettera tutti sono avidi di sentirla': passato e futuro dell'epistolario di Cesarotti." Giornale Storico della Letteratura Italiana 187. 617 (2010): 108-117.

Fantato, Michela. L'epistolario 'veneto' di Melchiorre Cesarotti: edizione critica e commento. Tesi di dottorato, Dipartimento di Italianistica e Filologia romanza, I ciclo n. s., Università Ca’ Foscari di Venezia, 2002-2003.

"La dissimulazione onesta: il carteggio Cesarotti-Pagani Cesa." Quaderni veneti 42 (dicembre 2005): 119-177.

(a cura di). Parleremo allora di cose, di persone, di libri. Lettere di Melchiorre Cesarotti a Francesco Rizzo Patarol. Venezia: Istituto Veneto di Scienze Lettere ed Arti, 2006.

. "Melchiorre Cesarotti-Giuseppe Urbano Pagani Cesa: integrazione al carteggio (1781-1808)." Quaderni veneti 47-48 (gennaio-dicembre 2008): 143-188. 
. "Per il censimento e l'edizione dei carteggi di Melchiorre Cesarotti. Parte I. Storia e fortuna dell'Epistolario." Studi sul Settecento e l'Ottocento 7 (2012): 121-130.

Gallo, Valentina. Cesarotti da Padova a Selvazzano. Padova: Provincia di Padova-Circolo amici della vecchia Selvazzano, 2008.

. "Gli autografi cesarottiani della Biblioteca Riccardiana di Firenze (mss. 3565-3566)." Critica letteraria 36. 4141 (2008): 645-675.

Frassineti, Luca. "Ricezione del soprannaturale in Cesarotti traduttore di Voltaire”. In Barbarisi e Carnazzi: 519-538.

Gilardino, Sergio Maria. "Melchiorre Cesarotti". In Epistolari e carteggi del Settecento. Edizioni e ricerche in corso. A cura di Antonio Postigliola. Con la collaborazione di Gennaro Barbarisi e Nadia Boccara. Roma: s.n., 1985: 32-34.

Melchiorre Cesarotti nel bicentenario della morte: 1808-2008. A cura della Scuola Secondaria di Primo grado "T. Albinoni” — Selvazzano Dentro [PD], Tipografia Italgraf, 2008.

Voltaire. Correspondence and Related Documents. A cura di Theodore Besterman. 51 voll. Oxford: Voltaire Fondation at the Taylor Institution, 1968-1977. 
